\title{
Uncertainty Analysis of the Representation of Air-Handling Unit with Terminal VAV Boxes in EnergyPlus
}

\author{
Qinpeng Wang ${ }^{1,2}$, Godfried Augenbroe ${ }^{2}$ \\ ${ }^{1}$ Siemens Smart Infrastructure, Buffalo Grove, USA \\ ${ }^{2}$ Georgia Institute of Technology, Atlanta, Georgia
}

\begin{abstract}
Over the past two decades, probabilistic building energy models are gaining popularity due to the recognition that many sources of uncertainty reside in our models. Most of previous uncertainty analysis research focuses on climate conditions, and building architectural parameters, such as envelope properties, and internal loads. Few studies investigate the impact of HVAC system representation on building energy predictions. In this paper, we wish to investigate the potential model inadequacy of the simplified representation of an air handing unit with terminal VAV boxes in EnergyPlus. The major simplification of the typical models for above system is that EnergyPlus does not perform pressurebased simulations of the air loop. To capture such neglected physical behavior, we construct in parallel to the normal model, a high-fidelity model with Modelica. A comparative study of the low and high-fidelity model shows that for supply air flowrates, both approaches capture the day-to-day variability well, and the $N M B E$ of EnergyPlus is only $1.3 \%$. When it comes to the comparison of fan power, EnergyPlus results show significant discrepancy (NMBE of $41.5 \%$ ) with the highfidelity model results.
\end{abstract}

\section{Introduction}

In large commercial buildings, Heating, ventilation and air conditioning (HVAC) systems always represent the largest primary energy end use (Huang, Wang, Huang, \& Augenbroe, 2015). Therefore, the importance of truthfully representing HVAC systems in building performance simulation cannot be understated. Conventionally, more attention has focused on the characterization of uncertainty in building thermal parameters, rather than on HVAC system parameters. Our models of HVAC systems represent simplified and ideal behavior, whereas in reality the system may behave astray from idealization.

In current literature, few studies investigate the impact of HVAC system representation on building energy predictions. Sun, Gu, Wu, and Augenbroe (2014) propose a framework for HVAC system sizing, taking into account uncertainty associated with building thermal loads. Augenbroe et al. (2013) investigate whether the practice of modeling the HVAC system as an integral part of the building simulation is justifiable, in other words, whether the fully coupled dynamic approach could be replaced with an uncoupled sequential approach (i.e., using a dynamic building simulation followed by a simplified HVAC calculation). In their study, building parameter uncertainties as well as families of equipment performance curves reflecting manufacturing tolerances are incorporated. Rasouli, Ge, Simonson, and Besant (2013) evaluate the effectiveness and economic performance of energy recovery ventilators (ERVs) under arbitrary uncertainty associated with building and HVAC system input parameters. L. P. Wang, Mathew, and Pang (2012) study the combined effect of various building operation practice, such as HVAC equipment schedule, VAV box minimum flow setting, and room temperature set-points for occupied hours. However, most input parameters in their study are associated with parametric variations instead of rigorously quantified uncertainty range, for instance, minimum airflow fractions of $15 \%$, $30 \%$, and $50 \%$ of the maximum airflow are used to represent the range of practice. Another study by L. Wang (2014) identifies a sampled list of maintenance issues, including cooling tower fouling, boiler/chiller fouling, refrigerant over or under charge, and temperature sensor offset, etc., and study their energy impact for an office building. Huang et al. (2015) propose a method of estimating the aging effect of chillers with a Bayesian Markov Chain Monte Carlo method, which is probabilistic in nature compared to a conventional deterministic maintenance factor approach (aging related deterioration is 0.01 for systems that undergo annual professional maintenance, and 0.02 for those that are seldom maintained). Yan, Li, Malkawi, and Augenbroe (2017) analyse an HVACsim+ model along with field measurement data and identifies the inadequacy of the EnergyPlus model in representing one type of minimum outdoor air control in VAV systems with a fixed minimum damper position to ensure minimum outdoor air supply. The results show that when the damper is at a fixed minimum position, neither the outdoor airflow rate nor the outdoor air ratio is the assumed fixed value in actual systems, contrary to the model assumptions in most building energy simulation models, such as EnergyPlus.

In particular, this paper wishes to investigate the potential model inadequacy of the simplified representation of VAV systems in EnergyPlus. This type of system is chosen for investigation because of its wide application. Various research has investigated into VAV systems from different perspectives. Aynur, Hwang, and Radermacher (2009b) simulate a VAV system in an existing office building with EnergyPlus with carefully prepared input 
parameter values that match the reality, and they find $71 \%$ simulated power consumption data fall within $\pm 15 \%$ range from the measured data. In another study by Aynur, Hwang, and Radermacher (2009a), they compare VAV and variable refrigerant flow (VRF) systems in an existing office building environment and find that overall VRF systems deliver $27.1 \%$ to $57.9 \%$ energy savings in regard to VAV systems, depending on system configuration, indoor and outdoor conditions. Mei and Levermore (2002) fit a polynomial curve and artificial neural network model to represent the non-linear characteristics of a fan, and inset the fan and terminal box models into HVACSIM+. Yoshida, Kumar, and Morita (2001) propose recursive autoregressive exogenous algorithm as a dynamic fault detection model for VAV air handling units, and their results are validated with data obtained from a real building after introducing artificial faults. Yang, Jin, Du, Fan, and Chai (2011) compare four control strategies of VAV systems in terms of indoor air temperature, energy consumption, $\mathrm{CO}_{2}$ concentration and predicted mean vote in an office building in China. S. W. Wang and Jin (2000) propose an online model-based methodology for the optimal control of VAV systems. Looking through the literature, insufficient attention has been paid to the investigation of the accuracy of VAV system representation in whole-building energy simulation models. This study particularly aims at potential inadequacy of the EnergyPlus representation of an air-handling unit (with variable speed fans) connected with terminal VAV boxes (with reheat capability).

\section{Methods}

First, we present a schematic of the VAV system. In order to quantify the impact of such simplification, we construct a high-fidelity model that captures missing physical behavior. The next step will be quantifying if there is significant discrepancy between the low and high-fidelity models and whether the outcome of the high-fidelity model (or the discrepancy between both models) could be mapped onto existing parameters within EnergyPlus and represented by parameter uncertainty distributions. Eventually, we study how results can be generalized for uncertainty analysis of VAV systems.

\section{Schematic of an Air-Handling Unit with Terminal VAV Boxes}

We choose to study a variable air volume (VAV) flow system with economizer and a heating and cooling coil in the air handling unit, and there are terminal VAV boxes with reheat coils at each supply branch. This is the common setup of a VAV system, but the control sequence of the same system setup could lead to quite different implementations. For instance, ASHRAE (2005) suggests two control sequences for the chilled water coil/hot water coil/economizer system type, and there are in total 19 sequences for VAV systems depending on system configurations. In this study, we implement the control sequence VAV 2A2-21232. The system schematic along with control system objects are depicted in Figure 1. The air handling unit supply fan speed modulates to maintain duct static pressure set-point, while the return fan is regulated to track the flow rate of the supply fan. The duct static pressure is reset based on terminal VAV box demands so that at least one of the VAV boxes is at $90 \%$ open. The heating coil valve, mixed air dampers and cooling coil valve modulate and together maintain the supply air temperature. Economizer free cooling is enabled when outside air dry bulb temperature is less than the return air's minus a deadband. In each supply branch, the VAV damper is controlled to meet the room temperature set-point for cooling, or fully open during heating. The reheat coil is adjusted to track the room temperature set-point for heating.

\section{Description of the EnergyPlus Model and Its Potential Inadequacy}

The major simplification of an EnergyPlus (version 8.2) representation of the above system is that EnergyPlus does not perform pressure-based simulations of the air loop. Such simplification has several repercussions: first, all VAV boxes in EnergyPlus operate standalone, without interacting with other VAV boxes, while in reality, when one VAV damper opens or closes, it might impact the duct static pressure distribution as well as static pressure setpoint and therefore the fan speed, such that all remaining VAV dampers have to adjust accordingly. In the actual VAV system, all airflows are determined by the duct static pressure distribution and the performance curves of the fans. Second, since the system pressure that the fan is facing is not known, there is no way of computing the fan speed, which is required for computing the electrical power accurately using the fan similarity laws. In fact, the implementation of variable speed fan within EnergyPlus is quite simple:

$f_{\text {flow }}=\dot{m} / m_{\text {design }}$,

$f_{p l}=c_{1}+c_{2} \cdot f_{\text {flow }}+c_{3} \cdot f_{\text {flow }}^{2}+c_{4} \cdot f_{\text {flow }}^{3}+c_{5} \cdot f_{\text {flow }}^{4}$,

$Q_{\text {tot }}=\frac{f_{p l} \cdot m_{\text {design }} \cdot \Delta P}{\eta_{\text {tot }} \cdot \rho_{\text {air }}}$,

where $Q_{t o t}$ is the fan power in watts, $\dot{m}$ is the air mass in $\mathrm{kg} / \mathrm{s}, m_{\text {design }}$ is the design air flow in $\mathrm{kg} / \mathrm{s}, \Delta P$ is the fan design pressure rise in Pascals, $\eta_{\text {tot }}$ is the fan total efficiency, $f_{\text {flow }}$ is the flow fraction, $f_{p l}$ is the part load factor. Analyzing input parameters for the fan model, we realize that the polynomial curve that relates $f_{p l}$ with $f_{\text {flow }}$ is a matter of choice for the energy modeler. Table 1 below, from Wray and Matson (2003), suggests default coefficient values, but the exact condition under which they are derived may not match the situation in reality. In the study, the one for the generic variable speed drive is chosen as a baseline for further comparisons, since this curve is also used by the EnergPlus default HVAC template for VAV systems. The third deficiency is that there are no local controls such as proportional and proportional-integral controllers implemented in EnergyPlus. Instead, each branch or damper is operated in an idealized fashion, in the sense that the supply side is always able to deliver what is demanded subject to component capacity from sizing calculations, and that dampers are able to control airflow perfectly without any delay or fluctuations. 
Table 1 Polynomial coefficients for fan performance

\begin{tabular}{|c|c|c|c|c|c|}
\hline $\begin{array}{c}\text { Fan } \\
\text { Control } \\
\text { Type }\end{array}$ & C1 & C2 & C3 & C4 & C5 \\
\hline $\begin{array}{c}\text { Outlet } \\
\text { Damper }\end{array}$ & 0.3507 & 0.3085 & -0.5414 & 0.8720 & 0 \\
\hline $\begin{array}{c}\text { Inlet } \\
\text { Vane }\end{array}$ & 0.3707 & 0.9725 & -0.3424 & 0 & 0 \\
\hline $\begin{array}{c}\text { VSD } \\
\text { (Generic) }\end{array}$ & 0.0015 & 0.00521 & 1.1086 & -0.1164 & 0 \\
\hline $\begin{array}{c}\text { VSD } \\
\text { (Title 24) }\end{array}$ & 0.1021 & -0.1177 & 0.2647 & 0.7600 & 0 \\
\hline
\end{tabular}

\section{Description of the High-Fidelity Model}

We adapt a Modelica implementation of a VAV system from the Modelica Buildings library (Wetter, Zuo, Nouidui, \& Pang, 2014) and apply to this study. Modelica is an equation-based object-oriented modeling language that specializes in describing and analyzing complex systems represented by differential, algebraic and discrete equations. The Buildings library is developed by LBNL to support rapid prototyping of innovative design and control of building systems, and has been widely accepted as of high fidelity. In the Modelica model of the VAV system, we capture actual control sequences, in the sense that the control input is always a physical quantify measured by a sensor, compared to an idealized thermal load customary in many building simulation programs including EnergyPlus. In addition, the control output is an actual control signal, such as the damper or valve position, contrary to flow rate in EnergyPlus. Therefore, the Modelica model can accurately represent air flowrate at a given VAV box as not only a function of its own damper position, but also of the damper characteristics, upper stream fan speed, fan curve, and the entire duct distribution network including other VAV box dampers. In this study, we implement a forward-curved fan from Carrier. Figure 2 (upper) shows the fan performance curves at the maximum and minimum speeds, normalized by the design airflow rate on the $\mathrm{X}$-axis, and the design static pressure on the Y-axis. Figure 2 (lower) shows normalized fan total efficiency (with regard to maximum efficiency at 0.611) against normalized airflow rate. During the simulation, once the control output in terms of normalized fan speed $r_{N}$ ( 0 to 1 ) has been determined by the controller, the new fan operating point is governed by the following equation that relates fan static pressure with volume flow rate:

$\Delta P=r_{N}^{2} \cdot s\left(\frac{\dot{V}}{r_{N}}, d\right)-\Delta P_{\varepsilon}$,

where $\dot{V}$ is the volume flow rate in $\mathrm{m}^{3} / \mathrm{s}, s$ is a cubic hermite spline that interpolates input data points $d$ on the performance curve with $r_{N}=1$.

Similarly, the following equation computes fan efficiency based on fan speed and volume flow rate:

$\eta=\eta_{\text {max }} \cdot s\left(\frac{\dot{v}}{r_{N}}, d\right)$,

where $s$ is a cubic hermite spline that interpolates input data points $d$ on the efficiency curve with $r_{N}=1, \eta_{\max }$ is the maximum fan total efficiency.
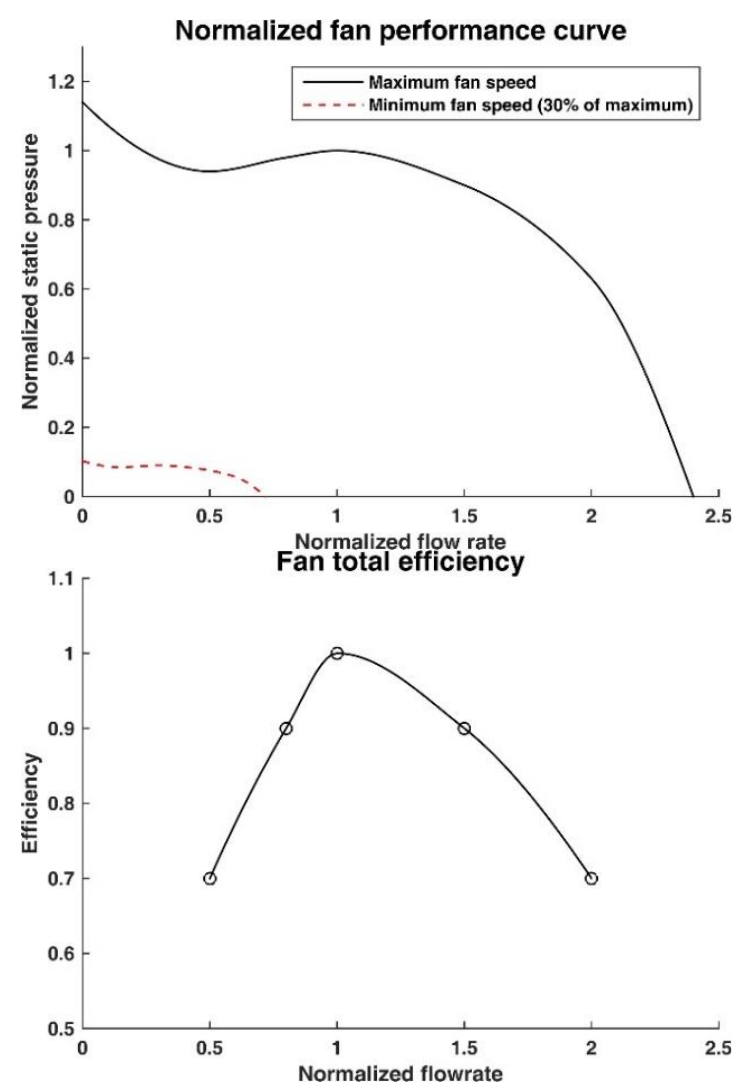

Figure 2 Fan characteristics in this study

When the fan pressure, flowrate and efficiency are all determined, Equation 1 is also used for computing fan power consumptions. The static pressure loss when the air stream flows through ducts can be described with a resistance model with a fixed flow coefficient, governed by the following equation:

$\dot{m}=k \sqrt{\Delta P}$,

where $k$ is a constant flow coefficient in $\sqrt{\mathrm{kg} \cdot \mathrm{m}}$.

In contrast, the flow resistance of a damper varies with the damper position. In this study, we implement a damper model from ASHRAE 825-RP (Haves, Norford, DeSimone, \& Mei, 1996) with its flow coefficient an exponential function of the opening angle. Damper flow resistance can be represented by:

$k_{d}(y)=\exp (a+b(1-y))$,

where $k_{d}$ is the damper characteristics, $a$ and $b$ are constants, $y$ is damper position, when $y=0$ corresponds to a closed damper, while $y=1$ means the damper is open. The above equation only applies when damper position is within range bounded by $\left(y_{L}, y_{U}\right)$. Outside this range, $k_{d}$ is defined by a quadratic polynomial that matches the damper resistance at $y=y_{L}$ and $y=0$, or $y=y_{U}$ and $y=1$, respectively. The flow coefficient of the damper can be calculated as:

$k(y)=\sqrt{\frac{2 \rho}{k_{d}(y)}} A$,

where $A$ is the face area of the damper.

Since the resistance of a VAV box is a combination of a fixed-resistance component and a time varying one, we 
combine Equation 5 and 6 and derive the flow coefficient for a VAV box as $k_{e f f}=\frac{k_{f i x} \cdot k(y)}{\sqrt{k_{f i x}^{2}+k^{2}(y)}}$, where $k_{e f f}$ is the

effective flow coefficient.

\section{Co-simulation Configuration}

Now that we have implemented a Modelica representation of the VAV system, we are ready to investigate the potential inadequacy of the EnergyPlus VAV model. We take as a case study one floor from the new construction medium office building in Atlanta from the DOE reference building pool (Deru et al., 2011), which consists of a core zone and four perimeter zones (southern, eastern, northern and western zones). Preparation for a cosimulation of EnergyPlus building and Modelica HVAC system takes the following three steps: configuring the EnergyPlus model for co-simulation (Nouidui, 2014), exporting the EnergyPlus building as a Functional MockUp Unit (FMU), configuring the Modelica model for cosimulation. An FMU is a simulator or model that supports co-simulation according to Functional Mock-Up Interface (Pazold, Burhenne, Radon, Herkel, \& Antretter, 2012), which is a tool-independent standard for model exchange and co-simulation of dynamic models. The role of the EnergyPlus model is to capture the thermal response of the room resulting from supply and return airflow by the Modelica HVAC system, and provide feedback to the Modelica model with room air temperatures. Therefore, in EnergyPlus, we create "OtherEquipment" objects associated with schedule values being the sensible and latent heat gain resulting from the supply air flowing into the room boundary and the return air flowing out. During the co-simulation, the Modelica model will dynamically change these schedule values and feed into EnergyPlus, while the latter will output room air temperatures to be read by Modelica controllers. A simplified configuration of EnergyPlus with schedules is shown in Figure 3.

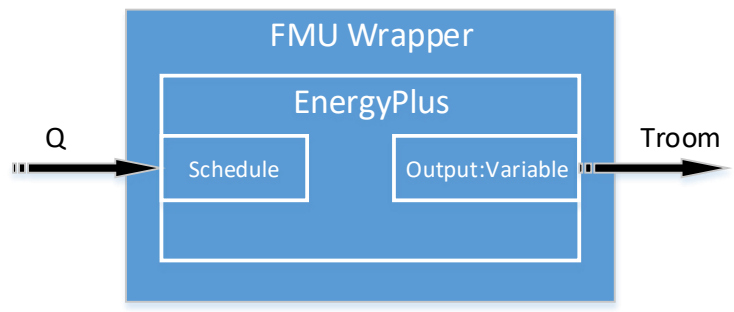

Figure 3 Configuration of EnergyPlus with schedules

For exporting the EnergyPlus model as an FMU, we adopt the utility "EnergyPlusToFMU", developed by LBNL (LBNL Simulation Research Group, 2016). Now we have a system with two simulation programs: EnergyPlus being the slave program packaged as an FMU for co-simulation, and Modelica as the master program that imports the FMU. For the detailed data exchange algorithm, readers are referred to descriptions in Q. Wang (2016).

Recall that the EnergyPlus FMU contains only the building model without HVAC systems, so the Modelica HVAC model cannot export the amount of supply and return airflow to EnergyPlus. Instead, we need to compute within Modelica the amount of sensible and latent heat flow into each room boundary by considering both supply and return air streams. In addition, since the medium office building does not perform a pressure-based airflow network simulation, the coupling scheme in our cosimulation ignores the pressure disturbance to the room by HVAC airflows as well as infiltration and inter-zone air flows. A specialized Modelica component is created to represent the return air stream from each room. Such a component takes as input the mass flowrate, air temperature, and relative humidity and outputs the sensible and latent enthalpy flowrate of the air streams with properties of the return air from each room, as depicted in Figure 4.

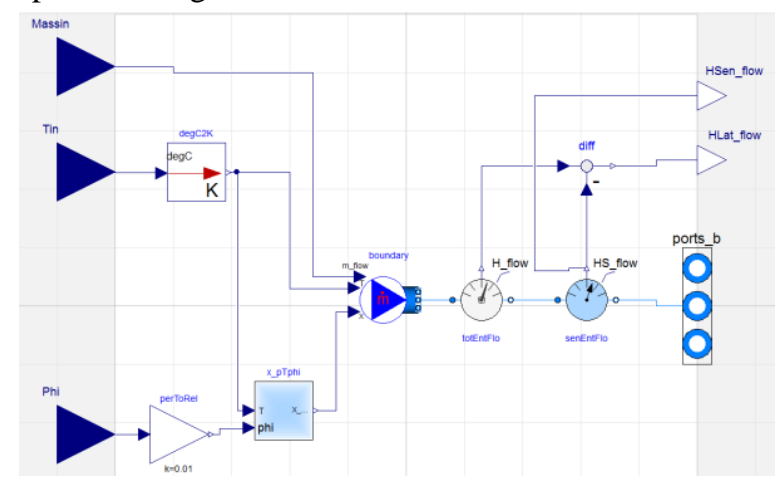

Figure 4 A specialized component in Modelica

The key part of this component is the "Buildings.Fluid.Sources.MassFlowSource_T" from the Buildings library, which produces a prescribed mass flow with prescribed temperature and mass fraction. The overall Modelica model is shown in Figure 5. Since the Modelica model does not auto-size, we take design supply airflows for each zone from EnergyPlus sizing results and input into Modelica. In return, we take the fan operating pressure that corresponds to the HVAC ductwork and use it for EnergyPlus fan consumption calculation $(\Delta P)$ in Equation 1.

\section{High-fidelity Model Preliminary Result}

Before uncertainty quantification of the EnergyPlus model, we demonstrate that the high-fidelity cosimulation is able to capture physical behavior neglected in the EnergyPlus model.

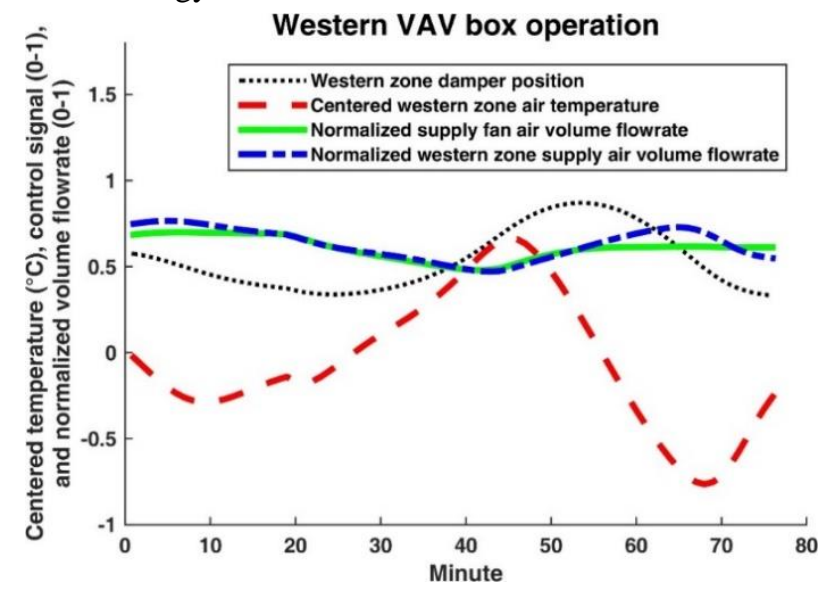

Figure 6 West zone VAV box operation 
In Figure 6 that depicts a sunny afternoon on July $5^{\text {th }}$, air volume flowrates are normalized against design airflow rates, rooms temperatures are centered around the cooling set-point at $24{ }^{\circ} \mathrm{C}$, and zone damper position is always between zero and one. We find that from around Minute 28 , the west room suffers from overheating, so the PI controller of the VAV box asks for more supply air into the zone. However, the ambient air is sufficiently cool such that all other zones demand less supply air, so the supply fan reduces its speed and overall supply air flowrate keeps decreasing. Consequently, the west zone is not supplied with sufficient supply air despite the increasing demand, and the room air temperature overshoots $0.7{ }^{\circ} \mathrm{C}$ above the set-point until Minute 45 . Such interactions cannot be captured by the EnergyPlus model since it assumes each VAV branch immediately receives the desired amount of supply air as long as the whole system capacity is not exceeded.

\section{Results}

Now we compare the simulation results of both platforms for the whole month of July in Atlanta in Figure 7. On all days but July 28, the temperature profile predictions for the core zone from both EnergyPlus and Modelica models follow almost the same trend, except that the temperatures from the Modelica model fluctuate around the set-point of $24{ }^{\circ} \mathrm{C}$ resulting from the imperfect control. Normalized mean bias error $\left(N M B E=\frac{\sum_{1}^{n}\left(y_{i}-\widehat{\left.l_{l}\right)}\right)}{(n-p) \cdot \bar{y}}\right)$ of the EnergyPlus model is only $0.14 \%$ if we regard Modelica results as a high-fidelity baseline. In terms of supply air flowrates in Figure 8, both platforms capture the day-to-day variability well, and the $N M B E$ of EnergyPlus is only $1.3 \%$. From the previous realization that EnergyPlus system representation has neglected key issues of physical behavior of the actual system, one might find this result puzzling. However, this is expected if we realize that both HVAC systems deliver supply air at the same state into the same building, and the room temperature responses show little discrepancy, so the predictions for supply air flowrates from both platforms should match well. The within-day fluctuations observed in the high-fidelity model are the results of VAV box interactions and imperfect control, which do not play a role in aggregated metrics such as $N M B E$. When it comes to the comparison of fan power in Figure 9, EnergyPlus results show significant discrepancy with the high-fidelity model results. Causes of such discrepancy are twofold: first, EnergyPlus simulates neither the fan speed nor the efficiency fluctuations with the varying load; second, EnergyPlus adopts the default polynomial curve that relates $f_{p l}$ with $f_{\text {flow }}$. In this case, EnergyPlus has a $N M B E$ of $41.5 \%$

We have thus established the inadequacy of EnergyPlus for predicting fan power accurately for the type of VAV system we are studying. In order to quantify this as an uncertainty in the low-fidelity model, we choose to map the high-fidelity model to existing parameters from EnergyPlus. To do so, we perform a least square polynomial curve fitting to normalized fan power (relative to fan power at design condition) against normalized volume flowrates from the Modelica model. Results have a fitting $R^{2}$ is 0.974 . The fitted polynomial coefficients are $(0,0.2169,-1.186,3.976,-1.978)$. As mentioned previously, this curve fit subsumes the combined effect of fan model simplification and imperfect control. If we rerun the EnergyPlus model with the updated coefficients, we achieve a much lower NMBE of $-1.37 \%$.

\section{Parametric Study}

So far, we have attempted the quantification of uncertainty associated with the EnergyPlus model of VAV systems. We know the resultant polynomial curve at least applies to the particular medium office building in Atlanta and the particular ductwork network in the Modelica model. However, in order to reach conclusions that are more general, we conduct parametric variations to the current case and analyze results. The first variation is to change the building characteristics. We update the building thermal model with higher internal loads, subject to hotter weather, i.e. in Phoenix. Since the thermal demand of the building changes, we perform another sizing calculation in EnergyPlus and update design fan pressure rise according to a Modelica HVAC simulation. However, the normalized fan performance curve and system resistance characteristics remain the same. Results for the fan power comparison present an excellent match. The $N M B E$ is only $-0.89 \%$. The second variation is to change the system resistance characteristics. To study this, we shrink the ductwork and damper flow coefficient $k$ to be $80 \%$ of the original assumptions, so effectively the fan is now facing a ductwork network with higher resistance resulting from higher friction loss and smaller face areas at the VAV dampers. Again, the airflow sizing calculation and fan sizing simulations are conducted to make sure the fan delivers design airflow rate at design pressure rise in Atlanta weather. Together with previous simulation runs, only the normalized fan performance curve always remains intact. Results for the fan power comparison again show a great match. The $N M B E$ is $1.72 \%$. The lesson from these two additional parametric studies is that the previously derived polynomial fit seems to apply to buildings served by VAV systems associated with similar normalized fan performance curves and control sequence to the one we study.

\section{Conclusions}

This paper presents model inadequacy of the simplified representation of an air handing unit with terminal vav boxes in EnergyPlus: blindly applying the default coefficients can lead to a $N M B E$ of $41.5 \%$ for fan energy, compared with a high-fidelity model in Modelica. This result applies to forward-curved fans in VAV system similar to the ones in our study in terms of normalized performance curve and control sequence. Future work is warranted where we apply the simulation framework in this paper to the analysis of more control sequences and system configurations. The outcome will be a repository of fan performance curves for future probabilistic energy simulations to sample from. Such uncertainty reflects both our ignorance of the HVAC system specification at 
design stage and the model simplification within EnergyPlus. Another potential improvement is to account for duct leakage in the high-fidelity model and observe the impact on the conclusion.

\section{References}

ASHRAE. (2005). Sequences of Operation for Common HVAC Systems.

Augenbroe, G., Zhang, Y., Khazaii, J., Su, H., Sun, Y., Lee, B. D., \& Wu, C. J. (2013). Implications of the uncoupling of building and HVAC simulation in the presence of parameter uncertainties. Paper presented at the Conference of International Building Performance Simulation Association, Chambery, France.

Aynur, T. N., Hwang, Y. H., \& Radermacher, R. (2009a). Simulation Comparison of VAV and VRF Air Conditioning Systems in an Existing Building for the Cooling Season. Energy and Buildings, 41(11), 11431150. doi:10.1016/j.enbuild.2009.05.011

Aynur, T. N., Hwang, Y. H., \& Radermacher, R. (2009b). Simulation of a VAV Air Conditioning System in an Existing Building for the Cooling Mode. Energy and Buildings, 41(9), 922-929. doi:10.1016/j.enbuild.2009.03.015

Deru, M., Field, K., Studer, D., Benne, K., Griffith, B., Torcellini, P., . . . Rosenberg, M. (2011). US Department of Energy Commercial Reference Building Models of the National Building Stock. Retrieved from

Haves, P., Norford, L. K., DeSimone, M., \& Mei, L. (1996). A Standard Simulation Testbed for the Evaluation of Control Algorithms and Strategies, RP825 Final Report. Retrieved from Atlanta:

Huang, P., Wang, Y., Huang, G., \& Augenbroe, G. (2015). Investigation of the Ageing Effect on Chiller Plant Maximum Cooling Capacity Using Bayesian Markov Chain Monte Carlo Method. Journal of Building Performance Simulation, 1-13. doi:10.1080/19401493.2015.1117529

LBNL Simulation Research Group. (2016). FMU Export of EnergyPlus.

Mei, L., \& Levermore, G. J. (2002). Simulation and Validation of a VAV System with an ANN Fan Model and a Non-Linear VAV Box Model. Building and Environment, 37(3), 277-284. doi:10.1016/s03601323(01)00028-2

Nouidui, T. S. (2014, August 26-28, 2013). Functional Mock-Up Unit Import in Energyplus for CoSimulation. Paper presented at the 13th Conference of International Building Performance Simulation Association, Chambéry, France.

Pazold, M., Burhenne, S., Radon, J., Herkel, S., \& Antretter, F. (2012, September 3-5, 2012). Integration of Modelica Models into an Existing Simulation Software Using FMI for Co-Simulation. Paper presented at the 9th International MODELICA Conference, Munich, Germany.

Rasouli, M., Ge, G. M., Simonson, C. J., \& Besant, R. W. (2013). Uncertainties in Energy and Economic Performance of HVAC Systems and Energy Recovery Ventilators Due to Uncertainties in Building and HVAC Parameters. Applied Thermal Engineering, $50(1)$, 732-742. doi:10.1016/j.applthermaleng.2012.08.021

Sun, Y. M., Gu, L., Wu, C. F. J., \& Augenbroe, G. (2014). Exploring HVAC System Sizing Under Uncertainty. Energy and Buildings, 81, 243-252. doi:10.1016/j.enbuild.2014.06.026

Wang, L. (2014). Modeling and Simulation of HVAC Faulty Operations and Performance Degradation Due to Maintenance Issues. Paper presented at the ASim 2012-1st Asia conference of International Building Performance Simulation Association, Shanghai, China, 11/25/12-11/27/12.

Wang, L. P., Mathew, P., \& Pang, X. F. (2012). Uncertainties in Energy Consumption Introduced by Building Operations and Weather for a Medium-Size Office Building. Energy and Buildings, 53, 152-158. doi:10.1016/j.enbuild.2012.06.017

Wang, Q. (2016). Accuracy, Validity and Relevance of Probabilistic Building Energy Models (Doctoral Dissertation). (Ph.D.)

Wang, S. W., \& Jin, X. Q. (2000). Model-Based Optimal Control of VAV Air-Conditioning System Using Genetic Algorithm. Building and Environment, 35(6), 471-487. doi:10.1016/s0360-1323(99)00032-3

Wetter, M., Zuo, W. D., Nouidui, T. S., \& Pang, X. F. (2014). Modelica Buildings Library. Journal of Building Performance Simulation, 7(4), 253-270. doi:10.1080/19401493.2013.765506

Wray, C. P., \& Matson, N. E. (2003). Duct Leakage Impacts on VAV System Performance in California Large Commercial Buildings. Lawrence Berkeley National Laboratory.

Yan, B., Li, X., Malkawi, A. M., \& Augenbroe, G. (2017). Quantifying uncertainty in outdoor air flow control and its impacts on building performance simulation and fault detection. Energy and Buildings, 134, 115128. doi:https://doi.org/10.1016/j.enbuild.2016.10.028

Yang, X. B., Jin, X. Q., Du, Z. M., Fan, B., \& Chai, X. F. (2011). Evaluation of Four Control Strategies for Building VAV Air-Conditioning Systems. Energy and Buildings, 43(2-3), 414-422. doi:10.1016/j.enbuild.2010.10.004

Yoshida, H., Kumar, S., \& Morita, Y. (2001). Online Fault Detection and Diagnosis in VAV Air Handling Unit by RARX Modeling. Energy and Buildings, 33(4), 391-401. doi:10.1016/s0378-7788(00)00121-3 


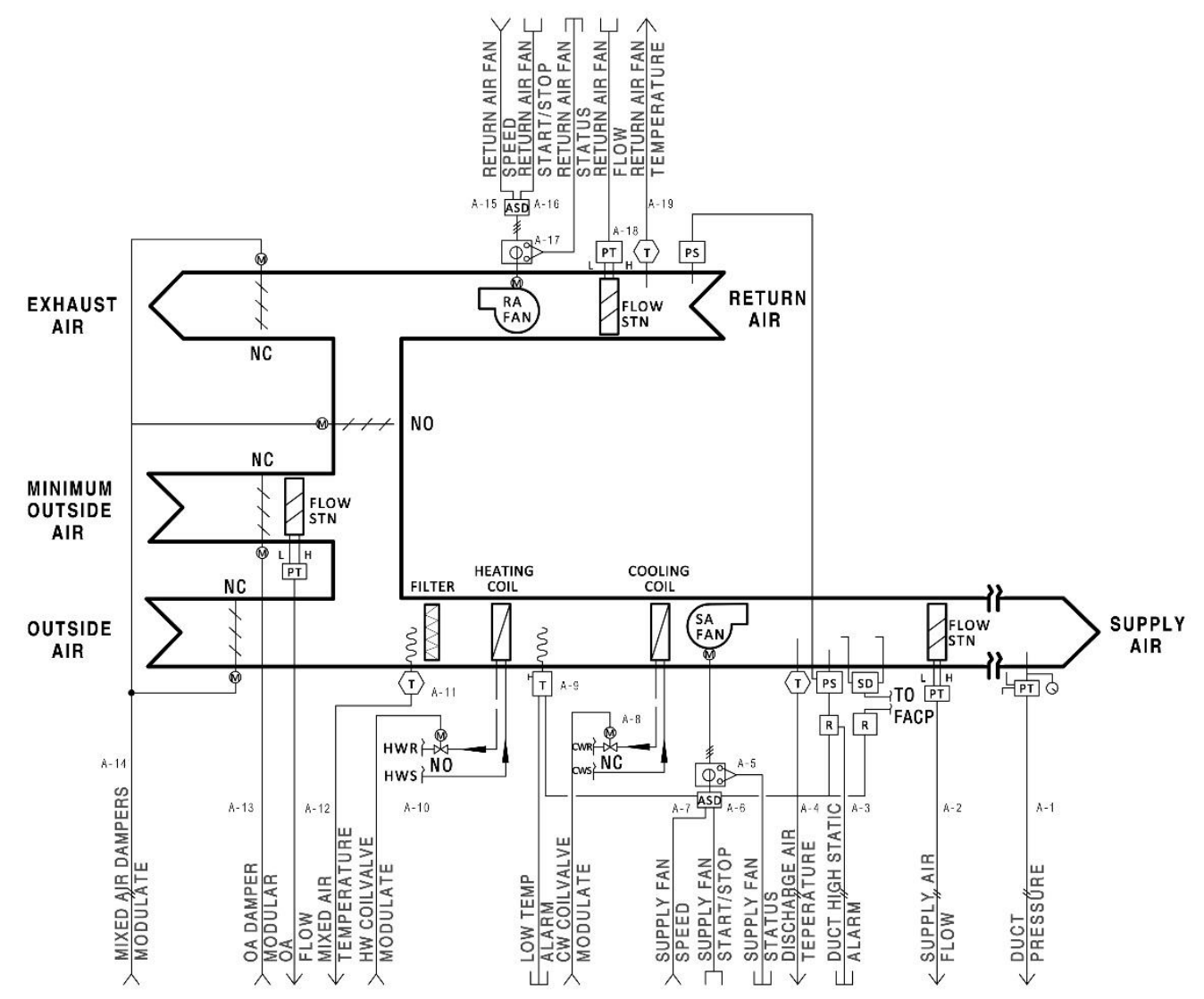

Figure 1 Schematic for control sequence VAV 2A2-21232 (Adapted from (ASHRAE, 2005))

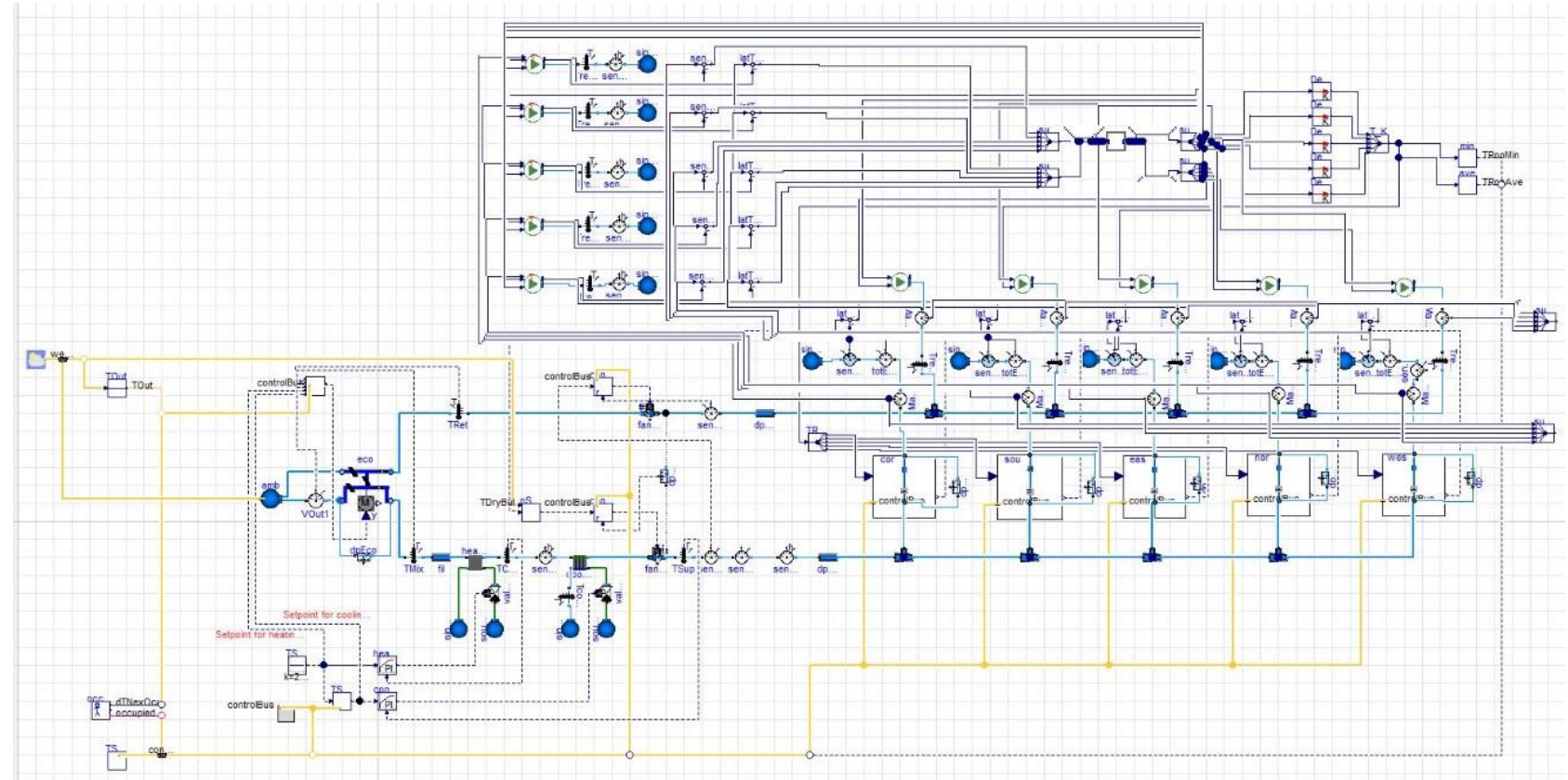

Figure 5 Overall Modelica model for the VAV system 


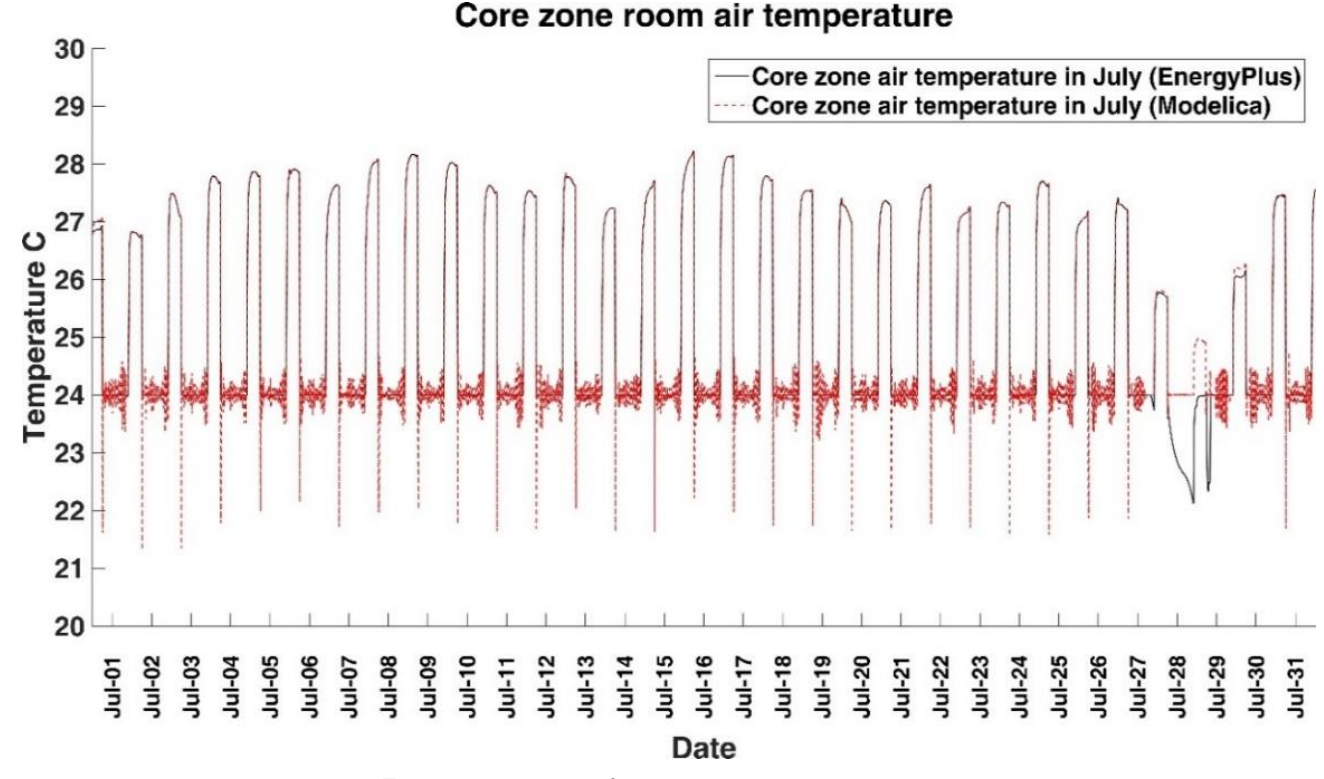

Figure 7 Comparison of core zone room air temperature

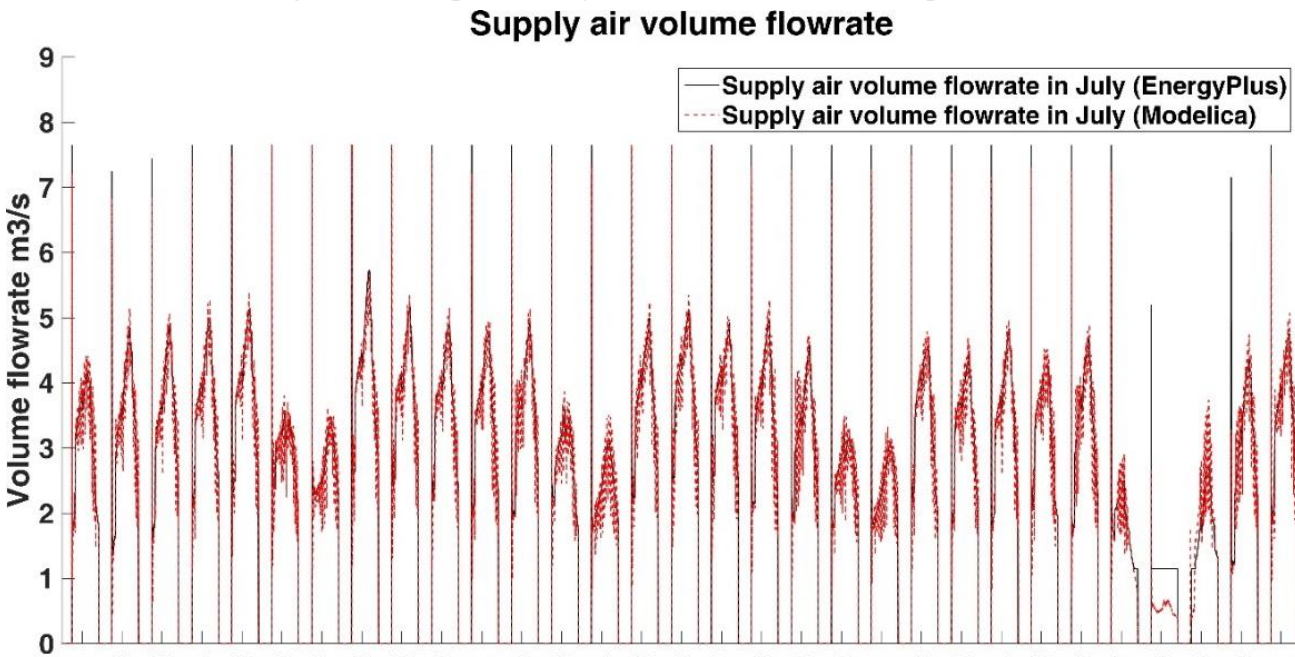

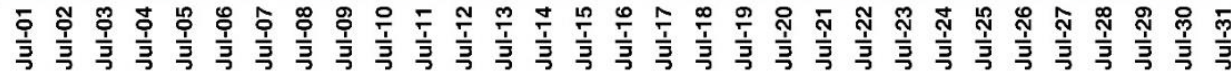
Date

Figure 8 Comparison of supply air volume flowrate

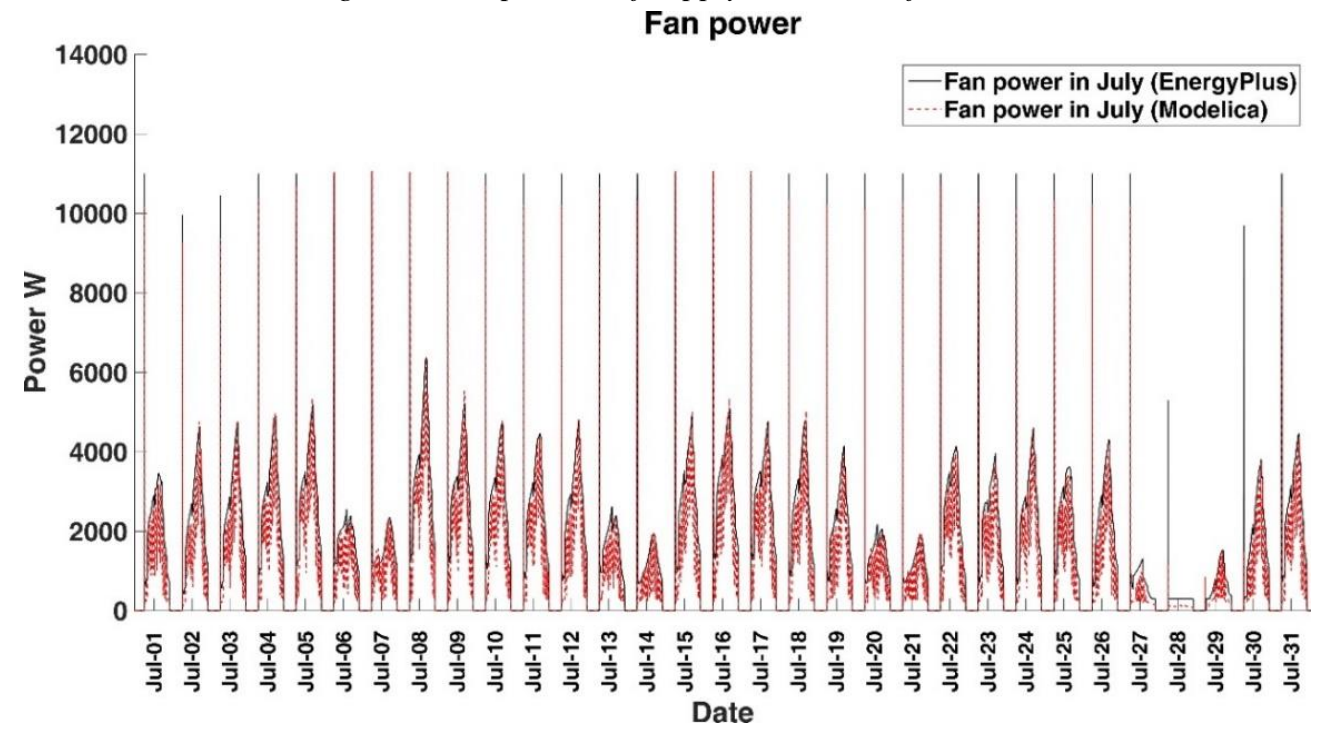

Figure 9 Comparison of fan power 